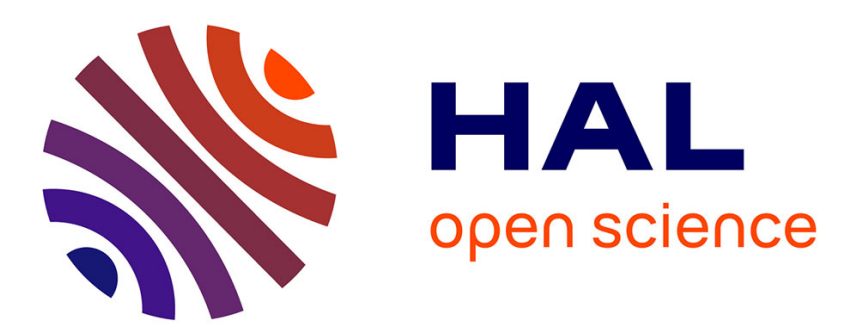

\title{
Extensions of networks with given diameter
}

\author{
Jean-Claude Bermond, Karima Berrada, J. Bond
}

\section{To cite this version:}

Jean-Claude Bermond, Karima Berrada, J. Bond. Extensions of networks with given diameter. Discrete Mathematics, 1989, 75 (1-3), pp.31-40. 10.1016/0012-365X(89)90075-7 . hal-02973248

\section{HAL Id: hal-02973248 \\ https://hal.inria.fr/hal-02973248}

Submitted on 21 Oct 2020

HAL is a multi-disciplinary open access archive for the deposit and dissemination of scientific research documents, whether they are published or not. The documents may come from teaching and research institutions in France or abroad, or from public or private research centers.
L'archive ouverte pluridisciplinaire HAL, est destinée au dépôt et à la diffusion de documents scientifiques de niveau recherche, publiés ou non, émanant des établissements d'enseignement et de recherche français ou étrangers, des laboratoires publics ou privés. 


\title{
EXTENSIONS OF NETWORKS WITH GIVEN DIAMETER
}

\author{
J.-C. BERMOND, K. BERRADA and J. BOND* \\ Laboratoire de Recherche en Informatique, Université Paris-Sud, Bât 490, 91405 Orsay Cedex, \\ France
}

\begin{abstract}
This article deals with combinatorial problems motivated by the design of large interconnection networks, in particular how to extend a network by adding nodes while keeping the degree and diameter small. We consider $D$-admissible extensions in which nodes are added one by one while the diameter remains constant. A $D$-admissible extension from a graph $G$ to a graph $G^{\prime}$ is a sequence of graphs $G=G_{0}, G_{1}, \ldots, G_{i}, \ldots, G_{k}=G^{\prime}$, where $G_{i}$ is a subgraph of $G_{i+1}$, $\left|V\left(G_{i+1}\right)\right|=\left|V\left(G_{i}\right)\right|+1$ and all of the $G_{i}$ have diameter at most $D$. Furthermore we insist that some of the $G_{i}$ are among the largest of the known graphs with maximum degree and diameter constant. We show that there exist $D$-admissible extensions from the hypercube of degree $d$ to the hypercube of degree $d+1$. Then we study $D$-admissible extension from the de Bruijn graph $\mathrm{UB}(d, D)$ [resp. Kautz graph $\mathrm{UK}(d, D)$ ] of maximum degree $2 d$ and diameter $D$ to $\mathrm{UB}(d+1, D)$ [resp. $\mathrm{UK}(d+1, D)]$, and show that such $D$-admissible extensions exist if $D=2$, but do not exist if $D>2$ and $d>4$.
\end{abstract}

\section{Introduction}

This article is motivated by some problems in the design of large interconnection networks. It is well known that such a network can be modelled by a graph in which the vertices represent the processors and the edges the links of the interconnection network. Various considerations and parameters play important roles in the design of such networks. For example one wants a small transmission delay in the network, which corresponds to a small diameter. In order to keep the cost low and to facilitate drawing one also wants a small number of links on each node, corresponding to a small maximum degree. Other constraints might be added (see the forthcoming book of D.I. Ameter and Max de Gree [1], or the surveys in $[2,4,9])$.

Here we emphasize the extendability properties of the network which are important, for example, for local area networks or communication networks. The number of processors in the network is not known in advance and ideally one wants to be able to add a new processor at any time while maintaining the desired properties of the network. For example, the designer might want to keep a small diameter and a small maximum degree. However, a graph with given maximum degree and diameter has a limit on the number of vertices, namely the Moore bound. Therefore it is not possible to add processors indefinitely while keeping the maximum degree and diameter fixed. Different classes of extensions can be considered according to the constraints imposed by the designer (for example

* Research partially supported by P.R.C. C3. 
"atomic" extensions if the processors are to be added one by one), and whether "relinkage" is allowed or not. "Relinkage" consists of deleting some links before adding vertices. This operation might be necessary; for example if one wants to keep the degree at most $\Delta$ and the original graph is $\Delta$-regular. In this case it is impossible to add a vertex and still have a connected graph without relinkage.

Finally let us note that the way we handle the problem will differ depending on the presence or absence of a bound on the size of the networks. If an upper bound is given for the maximum number of vertices of the network, it might be better to choose a "good" network having that size (the goal to be attained) and then to delete vertices to obtain different intermediate possible networks. This practical approach has been used in [5] for some graphs.

Here we consider infinite (or unbounded) extensions, where the vertices are to be added one by one (atomic extensions). We do not allow relinkage (strict extension) and want to keep the diameter $D$ constant. We will call such extensions $D$-admissible extensions. Other extensions have been considered by Bond and Konig [7] (fixed maximum degree) and by Konig [12] (fixed maximum degree and connectivity with or without relinkage). Before stating our results we present some definitions and notation.

\section{Definitions and notation}

The interconnection network will be modelled by an undirected graph $G=(V, E)$ where $V$ is the vertex set and $E$ the edge set. The degree of a vertex is the number of its neighbours and we will denote by $\Delta_{G}$ ( $\Delta$ if there is no ambiguity) the maximum degree of $G$. The distance $d_{G}(x, y)$ between two vertices $x$ and $y$ is the length of a shortest path between $x$ and $y$ in $G$. The diameter $D_{G}$ (or $D)$ is the maximum of $d_{G}(x, y)$ over all pairs of vertices $x$ and $y$ of $G$. $G$ will always be connected so $D_{G}$ is finite. A $(\Delta, D)$-graph will denote a graph with maximum degree $\Delta$ and diameter $D$.

Let $G$ and $G^{\prime}$ be two connected graphs. We will say that $G^{\prime}$ is a $D$-admissible extension of $G$ or that there exists a $D$-admissible extension from $G$ to $G^{\prime}$ if there exists a sequence of graphs $G=G_{0}, G_{1}, \ldots, G_{i}, \ldots, G_{k}=G^{\prime}$ such that

(i) $G_{i}$ is an induced subgraph of $G_{i+1}$,

(ii) $\left|V\left(G_{i+1}\right)\right|=\left|V\left(G_{i}\right)\right|+1$,

(iii) all the $G_{i}$ have diameter at most $D$.

Property (i) corresponds to adding vertices to $G$ without allowing relinkage. Property (ii) corresponds to adding the vertices one by one and property (iii) to keeping the diameter small during the process.

Furthermore, we want $G^{\prime}$ to be infinite or at least very large, and the $G_{i}$ to be "good" networks. The problem is that "good" is not well defined. One possibility is to insist that the $G_{i}$ have the smallest possible degree among all graphs with the same number of vertices and diameter $D$. However, the determination of this 
value is an unsolved problem. Another problem is that at some point one extension might be better (in a local sense) than another but so constrains us that we are forced to construct "worse" graphs later. To avoid these difficulties we restrict our attention to extensions such that at some steps the graphs obtained are among the "best" known (at least at the present time). More precisely we choose a family of graphs $G_{\Delta_{i}, D}$ such that $G=G_{\Delta_{1}, D} \subset G_{\Delta_{2}, D} \subset \cdots \subset G_{\Delta_{i}, D} \subset \cdots$ with $\Delta_{1} \leqslant \Delta_{2} \leqslant \cdots \leqslant \Delta_{i} \leqslant \cdots$ and we require that there exist $D$-admissible extensions from $G_{\Delta_{i}, D}$ to $G_{\Delta_{i+1}, D}$ for every $i$.

We first show that this is possible for the family of hypercubes (an interconnection currently used in parallel computers). Then we consider the best known general families of $(\Delta, D)$-graphs, namely the de Bruijn networks $\operatorname{UB}(d, D)$ or the Kautz networks UK $(d, D)$, both of which have maximum degree $2 d$ and diameter $D$. We show that for $D=2$ there exist $D$-admissible extensions from $\mathrm{UB}(d, 2)$ to $\mathrm{UB}(d+1,2)$, and count the number of nonisomorphic $D$-admissible extensions. Then we prove that for $D \geqslant 3$ and $d>4$ there do not exist $D$-admissible extensions from $\operatorname{UB}(d, D)$ to $\operatorname{UB}(d+1, D)$. Similar results are proved for Kautz networks.

\section{3. $D$-admissible extension of hypercubes}

The hypercube $\operatorname{CU}(d, D)$ has as vertices the words of length $D$ on an alphabet of $d$ letters, in other words the $D$-tuples $\left(a_{1}, a_{2}, \ldots, a_{D}\right)$ where $a_{i} \in$ $\{0,1, \ldots, d-1\}$. Two vertices are joined if their corresponding $D$-tuples differ in exactly one coordinate. When $d=2$, the hypercube $\operatorname{CU}(2, D)$ is the well known Boolean $D$-cube on $2^{D}$ vertices. The graph $\mathrm{CU}(d, D)$ is a regular graph with $d^{D}$ vertices, diameter $D$ and degree $(d-1) D$. Furthermore $\operatorname{CU}(d, D)$ is an induced subgraph of $\mathrm{CU}(d+1, D)$. This is a family as described above. Note that there exist different definitions for hypercubes, but the one above gives families with constant diameter.

Proposition 1. There exists a D-admissible extension from $\mathrm{CU}(d, D)$ to $\mathrm{CU}(d+1, D)$.

Proof. It suffices to add the vertices in the lexicographic order. Recall that the vertex $\left(a_{1}, a_{2}, \ldots, a_{D}\right)$ is lexicographically before $\left(b_{1}, b_{2}, \ldots, b_{D}\right)$ if for some $i$ we have $\left(a_{1}, a_{2}, \ldots, a_{i}\right)=\left(b_{1}, b_{2}, \ldots, b_{i}\right)$ and $a_{i+1}<b_{i+1}$. Suppose that at some step we have constructed a graph $G_{i}$, let $x$ be the first (in the lexicographic order) vertex of $\mathrm{CU}(d+1, D)$ which is not in $G_{i}$, and let $G_{i+1}$ be the spanning subgraph of $\mathrm{CU}(d+1, D)$ generated by the vertices of $G_{i}$ plus the vertex $x$. We have only to show that $G_{i}$ is of diameter $D$. That follows from the fact that in $\mathrm{CU}(d+1, D)$ between any pair of vertices there exists a monotonic path (in the lexicographic order). For example if $\left(a_{1}, a_{2}, \ldots, a_{D}\right)$ is before $\left(b_{1}, b_{2}, \ldots, b_{D}\right)$, the path (up 
to repetitions of some vertices) is

$$
\begin{array}{r}
\left(a_{1}, a_{2}, \ldots, a_{D}\right),\left(a_{1}, a_{2}, \ldots, a_{D-1}, b_{D}\right), \ldots, \\
\left(a_{1}, b_{2}, \ldots, b_{D}\right),\left(b_{1}, b_{2}, \ldots, b_{D}\right) .
\end{array}
$$

The hypercubes are interesting because they have nice properties like symmetry, easy routings, and high fault tolerance, but they are not among the best of the known $(\Delta, D)$-graphs. So it is interesting to study other families.

\section{D-admissible extension of de Bruijn graphs}

The de Bruijn digraph $B(d, D)$ (defined in [8]) with in- and out-degree $d$ and diameter $D$ is the digraph whose vertices are the words of length $D$ on an alphabet of $d$ letters $\{0,1, \ldots, d-1\}$. There is an arc from the vertex $x$ to a vertex $y$ if and only if the last $D-1$ letters of $x$ are the same as the first $D-1$ letters of $y$, that is there is an arc from $\left(a_{1}, a_{2}, \ldots, a_{D}\right)$ to the vertices $\left(a_{2}, \ldots, a_{D}, \lambda\right)$, where $\lambda$ is any letter of the alphabet. This digraph has $d^{D}$ vertices. We will denote by $\operatorname{UB}(d, D)$ the associated undirected de Bruijn graph of maximum degree $\Delta=2 d$ and diameter $D$. That is, $\operatorname{UB}(d, D)$ is the graph whose vertices are the words of length $D$ on an alphabet of $d$ letters in which the vertex $\left(a_{1}, \ldots, a_{D}\right)$ is adjacent to the vertices $\left(a_{2}, \ldots, a_{D}, \lambda\right)$ and $\left(\lambda, a_{1}, \ldots, a_{D-1}\right)$.

Proposition 2. $\mathrm{UB}(d, D)$ is an induced subgraph of $\mathrm{UB}(d+1, D)$.

Proof. It suffices to consider in $\operatorname{UB}(d+1, D)$ the vertices $\left(a_{1}, \ldots, a_{D}\right)$ such that $a_{i} \neq d$ for $1 \leqslant i \leqslant D$.

In view of the proposition above, one can try to construct a $D$-admissible extension from $\mathrm{UB}(d, D)$ to $\mathrm{UB}(d+1, D)$.

The case $D=1$ is trivial as $\operatorname{UB}(d, D)$ is the complete graph on $d$ vertices. In the case $D=2$ we have:

Proposition 3. There exists a $D$-admissible extension from $\mathrm{UB}(d, 2)$ to $\mathrm{UB}(d+1,2)$.

Proof. Let $G=\mathrm{UB}(d, 2)$. The vertices are the words $\left(a_{1}, a_{2}\right)$ with $a_{i} \in$ $\{0,1, \ldots, d-1\}$. We must add all of the $\left(a_{1}, a_{2}\right)$ 's such that at least one $a_{i}$ is $d$. We can do that in the following way: add first a vertex $(a, d)$ where $a \in\{0,1, \ldots, d-1\}$, then a vertex $(d, b)$ where $b \in\{0,1, \ldots, d-1\}$, then add all vertices containing exactly once the letter $d$ (in any order), and finally add $(d, d)$. 
In fact as we show in the next proposition, there exist many $D$-admissible extensions.

Proposition 4. The number of nonisomorphic D-admissible extensions from $\mathrm{UB}(d, 2)$ to $\mathrm{UB}(d+1,2)$ is $d ! N(d)$ where

$$
N(d)=\left(\begin{array}{c}
2 d \\
d
\end{array}\right)-\left(\begin{array}{c}
2 d-2 \\
d-1
\end{array}\right) \text {. }
$$

Proof. We have to add the $2 d-1$ vertices of $\operatorname{UB}(d+1,2)$ not in $\operatorname{UB}(d, 2)$, that is, the set $L \cup R \cup\{(d, d)\}$, where $L=\{(a, d) \mid 0 \leqslant a \leqslant d-1\}$ and $R=$ $\{(d, a) \mid 0 \leqslant a \leqslant d-1\}$.

Note that the vertex $(d, d)$ can be added only if all the vertices of $L$ or all the vertices of $R$ have already been added. Indeed, suppose $G_{i}$ contains the vertex $(d, d)$ but not the vertices $\left(a_{0}, d\right)$ and $\left(d, b_{0}\right)$. Then the distance in $G_{i}$ between $\left(b_{0}, a_{0}\right)$ (which is in $\left.\operatorname{UB}(d, 2)\right)$ and $(d, d)$ is 3 , and so $G_{i}$ does not have diameter 2 .

Note also that the first vertex to be added can be chosen to be in $L$, since $\mathrm{UB}(d+1,2)$ admits the symmetry $\sigma(a, b)=(b, a)$ as an automorphism. Then the second vertex must be in $R$. Indeed, if not suppose we add first $(a, d)$ and then $(b, d)$. Then in $G_{2}$ the distance between $(a, d)$ and $(b, d)$ is 3 .

Counting the number of nonisomorphic $D$-admissible extensions corresponds to counting the number of distinct ways of adding the vertices such that each step $G_{i}$ is of diameter 2. Two ways are distinct if there exists no automorphism of $\mathrm{UB}(d+1,2)$ mapping one extension on to the other.

Let $S(d)$ be the set of words of length $2 d+1$ on the alphabet $\{l, r, d\}$ containing the character $l d$ times, the character $r d$ times and exactly one $d$, such that each word begins with $l r$ and such that either all the l's or all the $r$ 's (or both) appear to the left of character $d$. To each $D$-admissible way of adding the vertices is associated a word of $S(d)$, built by putting an $l, r$ or $d$ in the $i$ th position depending on whether the $i$ th added vertex is in $L, R$ or is $(d, d)$. Conversely, each sequence corresponds to $d$ ! nonisomorphic ways of adding the vertices. Indeed we can decide that the vertices of $L$ are added in the order $(0, d),(1, d), \ldots,(d-1, d)$, since any permutation on the letters is an automorphism of $\operatorname{UB}(d+1,2)$. But then there are $d$ ! choices to associate to the characters $r$ vertices of $R$ which give nonisomorphic ways of adding the vertices.

Now it suffices to show that there are $N(d)$ words in $S(d)$. First let us count the number of words where the character $d$ appears after all the $l$ 's. That corresponds to choosing the positions of the $d-1$ occurrences $r$ among the last $2 d-1$ characters of the word. There are $\left(\begin{array}{c}2 d-1 \\ d-1\end{array}\right)$ such choices. Similarly there are $\left(\begin{array}{c}2 d-1 \\ d-1\end{array}\right)$ words in which the character $d$ appears after all the $r$ 's. Furthermore there are $\left(\begin{array}{c}2 d-2 \\ d-1\end{array}\right)$ words ending in $d$, thus

$$
N(d)=2\left(\begin{array}{c}
2 d-1 \\
d-1
\end{array}\right)-\left(\begin{array}{c}
2 d-2 \\
d-1
\end{array}\right)=\left(\begin{array}{c}
2 d \\
d
\end{array}\right)-\left(\begin{array}{c}
2 d-2 \\
d-1
\end{array}\right) \text {. }
$$


The case $D>2$

In view of the propositions above we thought that there were also many $D$-admissible extension from $\operatorname{UB}(d, D)$ to $\operatorname{UB}(d+1, D)$ for $D>2$. We tried different strategies to add vertices: for example, to add to $G_{i}$ a new vertex having the maximum number of neighbours in $G_{i}$. If there are many such vertices, add the one which is the smallest in the lexicographic order. Unfortunately none of the strategies worked. By using a branch and bound method we found a $D$-admissible extension from $\operatorname{UB}(2,3)$ to $\operatorname{UB}(3,3)$.

We give below an order in which to add the 19 new vertices containing the letter 2 .

$$
\begin{aligned}
& (0,0,2),(2,0,0),(0,1,2),(2,0,1),(1,2,0),(0,2,0),(1,0,2),(1,1,2), \\
& (2,1,0),(2,1,1),(0,2,1),(1,2,1),(2,0,2),(0,2,2),(2,2,0),(1,2,2), \\
& (2,1,2),(2,2,1),(2,2,2)
\end{aligned}
$$

Then we proved that at most 6 vertices can be added to $\operatorname{UB}(d, 3)$ without increasing the diameter if $d>2$. The proof can be found in [5]. Finally we proved the following.

Theorem 5. There does not exist a $D$-admissible extension from $\mathrm{UB}(d, D)$ to $\mathrm{UB}(d+1, D)$, where $D \geqslant 3$ and $d>4$.

Proof. Let $G_{0}=\mathrm{UB}(d, D)$ and suppose that there exists a $D$-admissible extension from $\mathrm{UB}(d, D)$ to $\mathrm{UB}(d+1, D)$, where $D \geqslant 3$ and $d>4$.

We will denote by $R(x)=\left\{\left(\lambda, a_{1}, \ldots, a_{D-1}\right)\right\}$ the neighbours of $x=$ $\left(a_{1}, a_{2}, \ldots, a_{D}\right)$ obtained by a right shift and by $L(x)=\left\{\left(a_{2}, \ldots, a_{D}, \mu\right)\right\}$ the neighbours of $\left(a_{1}, a_{2}, \ldots, a_{D}\right)$ obtained by a left shift. $L(x)$ corresponds to the successors of $x$ in the digraph $B(d, D)$ and $R(x)$ to its predecessors.

The proof will be split into two cases according to the parity of $D$.

Case 1. $D$ odd, $D=2 k+1(k \geqslant 1)$.

We will use the following proposition from $[3,6]$ :

Proposition 6. Let $a=\left(a_{1} a_{2} \cdots a_{D}\right)$ be a vertex of $\mathrm{UB}(d, D)$. Let the sets $Z_{l}(a)$ be defined for $l=1, \ldots, D$ as follows:

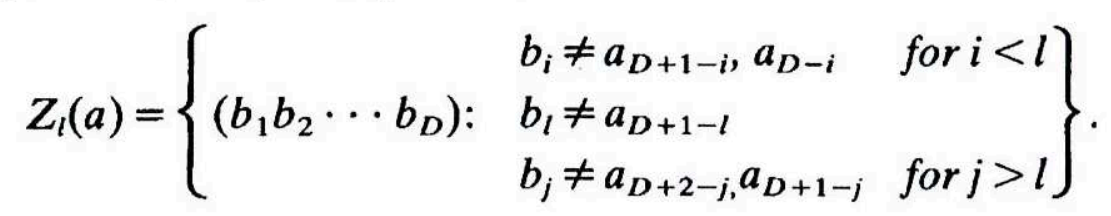

Let $Z(a)=\bigcup_{l=1}^{D} Z_{l}(a)$. Then, for every $b \in Z(a)$, the distance between $a$ and $b$ is $D$.

We will use the following corollary. 
Corollary 7. Let $S$ be a subset of the neighbours of a vertex $s$ in $\mathrm{UB}(d+1, D)$, such that $|S \cap R(s)| \leqslant d-3$ and $|S \cap L(s)| \leqslant d-3$. Then there exists a vertex $t=\left(t_{1}, \ldots, t_{D}\right)$ such that $t_{i} \neq d$ and $d(x, t)=D$ for every $x$ in $S$.

Proof. Let $r_{i}=\left(\lambda_{i}, s_{1}, \ldots, s_{D-1}\right)$ be the vertices of $S \cap R(s)$ and $l_{i}=$ $\left(s_{2}, \ldots, s_{D}, \mu_{i}\right)$ be the vertices of $S \cap L(s)(i=1, \ldots, d-3)$. We show that $Z=\left(\bigcap_{i=1}^{d-3} Z_{1}\left(r_{i}\right)\right) \cap\left(\bigcap_{i=1}^{d-3} Z_{D}\left(l_{i}\right)\right)$ contains a vertex $t=\left(t_{1}, \ldots, t_{D}\right)$ such that $t_{i} \neq k$. Note that

$$
Z=\left\{\begin{array}{ll}
b_{1} \neq \mu_{i}, s_{D}, s_{D-1} & \text { for } 1 \leqslant i \leqslant d-3 \\
\left(b_{1} b_{2} \cdots b_{D}\right): \begin{array}{ll}
b_{j} \neq s_{D+2-j}, s_{D+1-j}, s_{D-j} \\
b_{D} \neq s_{2}, s_{1}, \lambda_{i}
\end{array} & \text { for } 2 \leqslant j \leqslant D-1 \leqslant d-3
\end{array}\right\} .
$$

Thus we have at most $d-1$ constraints on the entries of the vertices in $Z$, so if we add the additional constraint $t_{i} \neq d$ the set is still not empty (we are in $\mathrm{UB}(d+1, D)$.

This proposition implies that before adding $s$ we must have already added either $d-2$ elements of $R(s)$ or of $L(s)$.

Now let us choose $s$ to be the first vertex added with a letter $d$ in the middle: $s=\left(a_{1}, \ldots, a_{k}, d, b_{1}, \ldots, b_{k}\right)$.

Without loss of generality suppose that when we add $s$ at least $d-2$ vertices of $R(s)$ have been added (the case $L(s)$ is identical) and let us denote these vertices $s_{i}=\left(\alpha_{i}, a_{1}, \ldots, a_{k}, d, b_{1}, \ldots, b_{k-1}\right)$, with $1 \leqslant i \leqslant d-2$. Let $G_{s}$ be the graph just before the adjunction of $s$. We will show that in $G_{s}$ there are two vertices $s_{i}$ and $s_{j}$ at distance greater than $D$. First of all, note that the distance between $s_{i}$ and any vertex of $G_{0}$ is at least $k$. Indeed, to reach a vertex of $G_{0}$ from $s_{i}$ we have to do at least $k$ right-shifts, as there does not exist in $G_{s}$ any vertex having $d$ in the middle. Now consider a shortest path in $G_{s}$ between $s_{i}$ and $s_{j}$. As $\alpha_{i} \neq \alpha_{j}$ and $a_{k} \neq d$ and there is no vertex with $d$ in the middle, this path is of the form $s_{i} \cdots z_{i} \cdots z_{j} \cdots s_{j}$, where $z_{i}$ and $z_{j}$ are in $G_{0}$ and $z_{i}=\left(*, \ldots, *, \alpha_{i}, a_{1}, \ldots, a_{k}\right)$, $z_{j}=\left(*, \ldots, *, \alpha_{j}, a_{1}, \ldots, a_{k}\right)$. As $d-2>2$ we can find $\alpha_{i}$ and $\alpha_{j}$ such that $\alpha_{i} \neq a_{1}, \alpha_{j} \neq a_{1}$, and therefore the distance between $z_{i}$ and $z_{j}$ is at least 2 . So the distance in $G_{s}$ between $s_{i}$ and $s_{j}$ is at least $2 k+2$, a contradiction.

Case 2. $D$ even $D=2 k+2(k \geqslant 1)$.

The proof is similar to case 1, but we use the following corollary of Proposition 6.

Corollary 8. Let $S$ be a subset of the neighbours of a vertex $s$ in $\mathrm{UB}(d+1, D)$, such that $|S| \leqslant d-2$. If there exist a vertex s such that $S \subset R(s)$ or $S \subset L(s)$ then there exists a vertex $t$ such that $t_{i} \neq d$ and $d(x, t)=D$ for every $x$ in $S$. 
Proof. Without loss of generality let us suppose $S \subset R(s)$. Let $r_{i}=$ $\left(\lambda_{i}, s_{1}, \ldots, s_{D-1}\right)$ be the vertices of $S(i=1, \ldots, d-2)$. We show that $Z=$ $\bigcap_{i=1}^{d-2} Z_{D}\left(r_{i}\right)$ contains a vertex $t=\left(t_{1}, \ldots, t_{D}\right)$ such that $t_{i} \neq k$. Note that

$$
Z=\left\{\begin{array}{lll} 
& b_{j} \neq s_{D-j}, s_{D-j-1} & \text { for } 1 \leqslant j \leqslant D-2 \\
\left(b_{1} b_{2} \cdots b_{D}\right): & b_{D-1} \neq \lambda_{i}, s_{1} & \text { for } 1 \leqslant i \leqslant d-2 \\
& b_{D} \neq \lambda_{i} & \text { for } 1 \leqslant i \leqslant d-2
\end{array}\right\} .
$$

This way we have at most $d-1$ constraints on the entries of the vertices in $Z$, so if we add the additional constraint $t_{i} \neq d$, the set is still not empty (we are in $\mathrm{UB}(d+1, D))$.

This proposition implies that before adding $s$ we must have added already either $d-1$ elements of $R(s)$ or $L(s)$.

Now let us choose $s$ to be the first vertex added with a letter $d$ in one of the two middle positions:

$s=\left(a_{1}, \ldots, a_{k}, x, d, b_{1}, \ldots, b_{k}\right)$ or $s=\left(a_{1}, \ldots, a_{k}, d, x, b_{1}, \ldots, b_{k}\right)$, with $x \neq d$.

Without loss of generality suppose that $s=\left(a_{1}, \ldots, a_{k}, x, d, b_{1}, \ldots, b_{k}\right)$, so that when we add $s$ at least $d-1$ vertices of $R(s)$ have been added (the case $L(s)$ is identical). Let these vertices be $s_{i}=\left(\alpha_{i}, a_{1}, \ldots, a_{k}, x, d, b_{1}, \ldots, b_{k-1}\right)$, with $1 \leqslant i \leqslant d-2$. Let $G_{s}$ be the graph just before the adjunction of $s$. We will show that in $G_{s}$ there are two vertices $s_{i}$ and $s_{j}$ at distance greater than $D$. First of all the distance between $s_{i}$ and any vertex of $G_{0}$ is at least $k$. Indeed to reach a vertex of $G_{0}$ from $s_{i}$ we have to do at least $k$ right-shifts, as there does not exist in $G_{s}$ any vertex having $d$ in the middle. Now consider a shortest path in $G_{s}$ between $s_{i}$ and $s_{j}$. As $\alpha_{i} \neq \alpha_{j}$ and $a_{k} \neq d$ and there is no vertex with $d$ in the middle, this path is of the form $s_{i} \cdots z_{i} \cdots z_{j} \cdots s_{j}$, where $z_{i}$ and $z_{j}$ are in $G_{0}$ and

$$
z_{i}=\left(*, \ldots, *, \alpha_{i}, a_{1}, \ldots, a_{k}, x\right), \quad z_{j}=\left(*, \ldots, *, \alpha_{j}, a_{1}, \ldots, a_{k}, x\right) .
$$

As $d-1>3$ we can find $\alpha_{i}$ and $\alpha_{j}$ such that $\alpha_{i} \neq a_{1}, \alpha_{j} \neq a_{1}, \alpha_{i} \neq a_{2}, \alpha_{j} \neq a_{2}$, and therefore the distance between $z_{i}$ and $z_{j}$ is greater than 2 . So the distance in $G_{s}$ between $s_{i}$ and $s_{j}$ is greater than $2 k+2$, a contradiction.

\section{Remarks}

(1) The proof does not give information on the number of vertices which can be added to $\operatorname{UB}(d, D)$ without increasing the diameter. It can be proved that this number is at least $2 d^{D-3}+4(d-1) d^{[D / 2]}+2 d$.

(2) An interesting problem is to give an extension from $\mathrm{UB}(d, D)$ to $\mathrm{UB}(d+1, D)$ such that all the $G_{i}$ have the smallest possible diameter, that is, to determine the smallest $D^{\prime}$ such that there exists a sequence $\operatorname{UB}(d, D)$, $G_{1}, \ldots, G_{i}, \ldots, \mathrm{UB}(d+1, D)$, with $G_{i}$ an induced subgraph of $G_{i+1},\left|V_{G_{i+1}}\right|=$ $\left|V_{G_{i}}\right|+1$ and all the $G_{i}$ having diameter at most $D^{\prime}$. It is easy to prove that $D^{\prime} \leqslant 3 D / 2$, but perhaps even $D^{\prime} \leqslant D+c$ holds for some constant $c$. 


\section{D-admissible extension of Kautz graphs}

Since the results are very similar for the other family of networks introduced by Kautz [11], we will not give proofs.

The undirected $\operatorname{Kautz}$ network $\operatorname{UK}(d, D)$ is the induced subgraph of the de Bruijn network $\mathrm{UB}(d+1, D)$ spanned by the vertices without two consecutive identical letters. So the vertices are labelled with words $\left(a_{1}, a_{2}, \ldots, a_{D}\right)$, where $a_{i}$ belongs to an alphabet of $d+1$ letters and $a_{i} \neq a_{i+1}$ for $1 \leqslant i \leqslant D-1$. The vertex $\left(a_{1}, a_{2}, \ldots, a_{D}\right)$ is joined to the $2 d$ vertices $\left(\lambda, a_{1}, \ldots, a_{D-1}\right)$ with $\lambda \neq a_{1}$ and $\left(a_{2}, \ldots, a_{D}, \mu\right)$ with $\mu \neq a_{D}$.

Thus $\operatorname{UK}(d, D)$ has $d^{D}+d^{D-1}$ vertices, maximum degree $2 d$ and diameter $D$.

Proposition 9. $\mathrm{UK}(d, D)$ is an induced subgraph of $\mathrm{UK}(d+1, D)$.

Therefore the $\operatorname{UK}(d, D)$ also form a good family of graphs to be considered for $D$-admissible extensions. They give rise to the following results, which are analogous to those for de Bruijn graphs.

Proposition 10. There exists a D-admissible extension from $\mathrm{UK}(d, 2)$ to $\mathrm{UK}(d+1,2)$.

In fact a proof similar to the one of Proposition 4 shows that there are $(d+1) !\left(\begin{array}{c}2 d \\ d\end{array}\right)$ such extensions.

Theorem 11. There does not exist a $D$-admissible extension from $\mathrm{UK}(d, D)$ to $\mathrm{UK}(d+1, D)$, where $D>2$ and $d>4$.

\section{Acknowledgement}

We thank C. Delorme and R. Kerjouan for stimulating discussions on this problem, and A.L. Liestman for his careful reading.

\section{References}

[1] D.I. Ameter and Max D. Gree, Graphs and Interconnection Networks, forthcoming book.

[2] J.-C. Bermond, J. Bond, M. Paoli and C. Peyrat, Graphs and interconnection networks: Diameter and vulnerability, in Surveys in Combinatorics, Invited Papers for the Ninth British Combinatorial Conf., 1-30, London Math. Soc. Lec. Note Ser. 82 (Cambridge University Press, 1983).

[3] J.-C. Bermond, J. Bond, S. Rudich, M. Santha and W.F. de la Vega, The radius of graphs on alphabets, submitted.

[4] J.-C. Bermond, C. Delorme and J.-J. Quisquater, Strategies for interconnection networks: some methods from graph theory, J. Parallel and Distributed Computing, 3, (1986) 433-449. 
[5] K. Berrada, Extension de réseaux d'interconnexion, Thése, Université de Paris Sud (1986).

[6] J. Bond, Grands réseaux d'interconnexion, Thése d'Etat, Université de Paris Sud (1987).

[7] J. Bond and J.-C. Konig, Extension de réseaux de degré maximum donnée, manuscript.

[8] N.G. de Bruijn, A combinatorial problem, Koninklijke Nederlandse Academie van Wetenschappen Proc., Ser A49 (1946) 758-764.

[9] F.R.K. Chung, Diameter of communication networks, AMS short course on the mathematics of information processing, Proceedings of Symposia in Applied Mathematics, 34, (AMS, Providence, 1986) 1-18.

[10] M.A. Fiol, J.L.A. Yebra and I. Alegre, Line digraph iterations and the $(d, k)$ digraph problem, IEEE Trans. on Computers, vol. C-33, (1984) 400-403.

[11] W.H. Kautz, Bounds on directed $(d, k)$ graphs, in Theory of Cellular Logic Networks and Machines, 20-28, AFCRL-68-0668, SRI Project 7528, Final report (1968).

[12] J.-C. Konig, Extensions de réseaux de connexité donnée, Proc. Coll. Combinatoire et Informatique (Montreal, May 1987) to appear. 